\title{
An Underlying Mechanism of Dual Wnt Inhibition and AMPK Activation: Mitochondrial Uncouplers Masquerading as Wnt Inhibitors
}

Wen Zhang ${ }^{1,2}$, Vitaliy M. Sviripa ${ }^{3,4}$, Liliia M. Kril ${ }^{1,2,3}$, Tianxin $\mathrm{Yu}^{1,2}$, Yanqi Xie ${ }^{1,2}$, W. Brad Hubbard ${ }^{5}$, Patrick G. Sullivan ${ }^{5}$, Xi Chen ${ }^{4}$, Chang-Guo Zhan ${ }^{4}$, Yang Yang-Hartwich ${ }^{6}$, B. Mark Evers ${ }^{2,7}$, Brett T. Spear ${ }^{2,8}$, Roberto Gedaly ${ }^{2,7,9}$, David S. Watt ${ }^{1,2,3^{*}}$ and Chunming Liu ${ }^{1,2^{*}}$

${ }^{1}$ Department of Molecular and Cellular Biochemistry, College of Medicine, University of Kentucky, Lexington, KY 40536

${ }^{2}$ Lucille Parker Markey Cancer Center, University of Kentucky, Lexington, KY 40536

${ }^{3}$ Center for Pharmaceutical Research and Innovation, College of Pharmacy, University of Kentucky, Lexington, KY 40536

${ }^{4}$ Department of Pharmaceutical Sciences, College of Pharmacy, University of Kentucky, Lexington, KY 40536

${ }^{5}$ Department of Neuroscience, College of Medicine, University of Kentucky, Lexington, KY 40536

${ }^{6}$ Department of Obstetrics, Gynecology and Reproductive Sciences, Yale School of Medicine LSOG 209, 375 Congress Avenue, New Haven CT 06510

${ }^{7}$ Department of Surgery, College of Medicine, University of Kentucky, Lexington, KY 40536

${ }^{8}$ Department of Microbiology, Immunology and Molecular Genetics, University of Kentucky, Lexington, KY 40536

${ }^{9}$ Transplant Center, College of Medicine, University of Kentucky, Lexington, KY 40536

*Correspondence to: chunming.liu@uky.edu ordwatt@uky.edu

\section{Table of Contents}

1) Fig. S1, HPLC trace for compound FTU-11

2) Fig. S2, HPLC trace for compound FDN-4E

3) Fig. S3, HPLC trace for compound FH535

4) Fig. S4, HPLC trace for compound FH535-M

5) Fig. S5, HPLC trace for compound Y3

6) Fig. S6, HPLC trace for compound Y3-M 
Fig. S1 HPLC trace for FTU-11
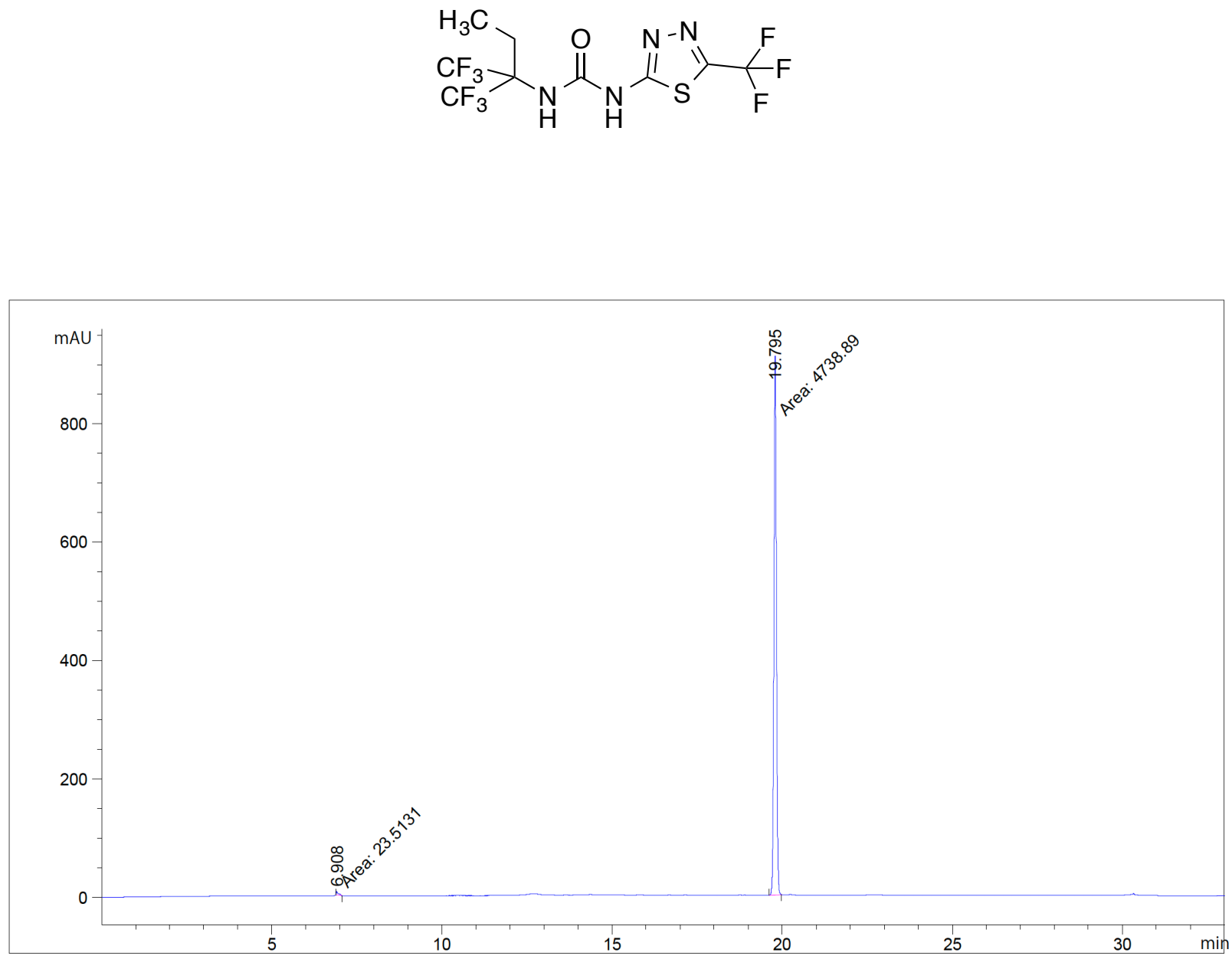

Peak RetTime Type Width Area Height Area

\# [min] [min] $\left[\mathrm{mAU}^{*} \mathrm{~s}\right] \quad[\mathrm{mAU}] \%$

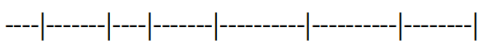

$\begin{array}{llllll}1 & 6.908 \mathrm{MM} & 0.0671 & 23.51305 & 5.84256 & 0.4937\end{array}$

$2 \quad 19.795$ MM $\quad 0.08674738 .88770910 .4757199 .5063$

Totals : $\quad 4762.40075916 .31826$ 
Fig. S2 HPLC trace for FND-4E
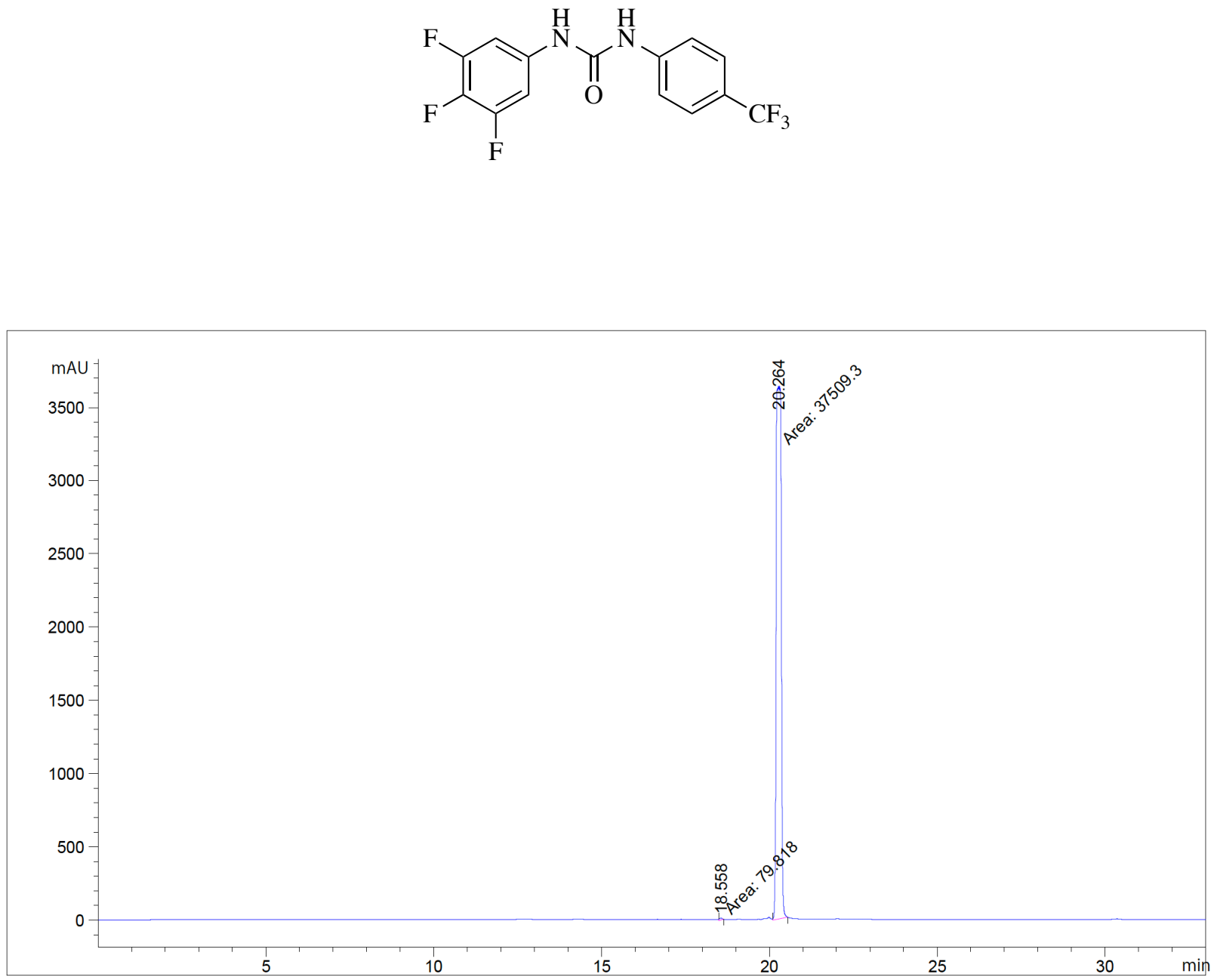

Signal 1: DAD1 A, Sig $=260,4$ Ref $=360,100$

Peak RetTime Type Width Area Height Area

\# [min] [min] $\left[\mathrm{mAU}^{*} \mathrm{~s}\right] \quad[\mathrm{mAU}] \%$

|

$\begin{array}{llllll}1 & 18.558 \mathrm{MM} & 0.0925 & 79.81796 & 14.37927 & 0.2123\end{array}$

2 20.264 MM $0.17183 .75093 \mathrm{e} 43638.9397099 .7877$

Totals : $\quad 3.75891 \mathrm{e} 4 \mathbf{3 6 5 3 . 3 1 8 9 6}$ 
Fig. S3 HPLC trace for FH535<smiles>Cc1cc([N+](=O)[O-])ccc1NS(=O)(=O)c1cc(Cl)ccc1Cl</smiles>

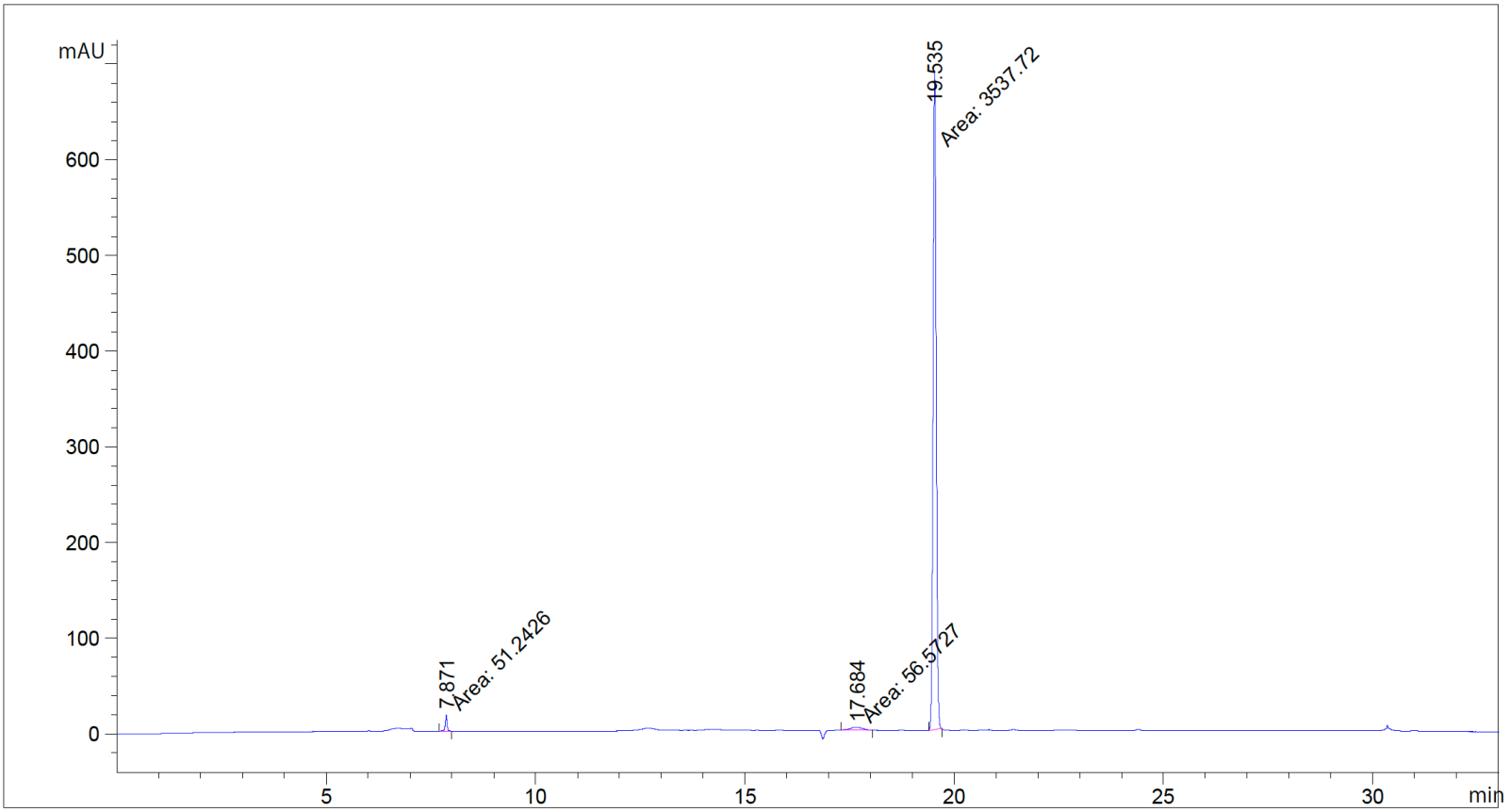

Signal 1: DAD1 A, Sig $=260,4$ Ref $=360,100$

Peak RetTime Type Width Area Height Area

\# [min] [min] [mAU*s] [mAU] \%

|

$\begin{array}{llllll}1 & 7.871 \mathrm{MM} & 0.0505 & 51.24261 & 16.90880 & 1.4056\end{array}$

$\begin{array}{llllll}2 & 17.684 \mathrm{MM} & 0.3477 & 56.57266 & 2.71185 & 1.5518\end{array}$

3 19.535 MM $\quad 0.08603537 .72046685 .6112197 .0425$

Totals : $\quad 3645.53573705 .23186$ 
Fig. S4 HPLC trace for FH535-M<smiles>Cc1cc([N+](=O)[O-])ccc1N(C)S(=O)(=O)c1cc(Cl)ccc1Cl</smiles>

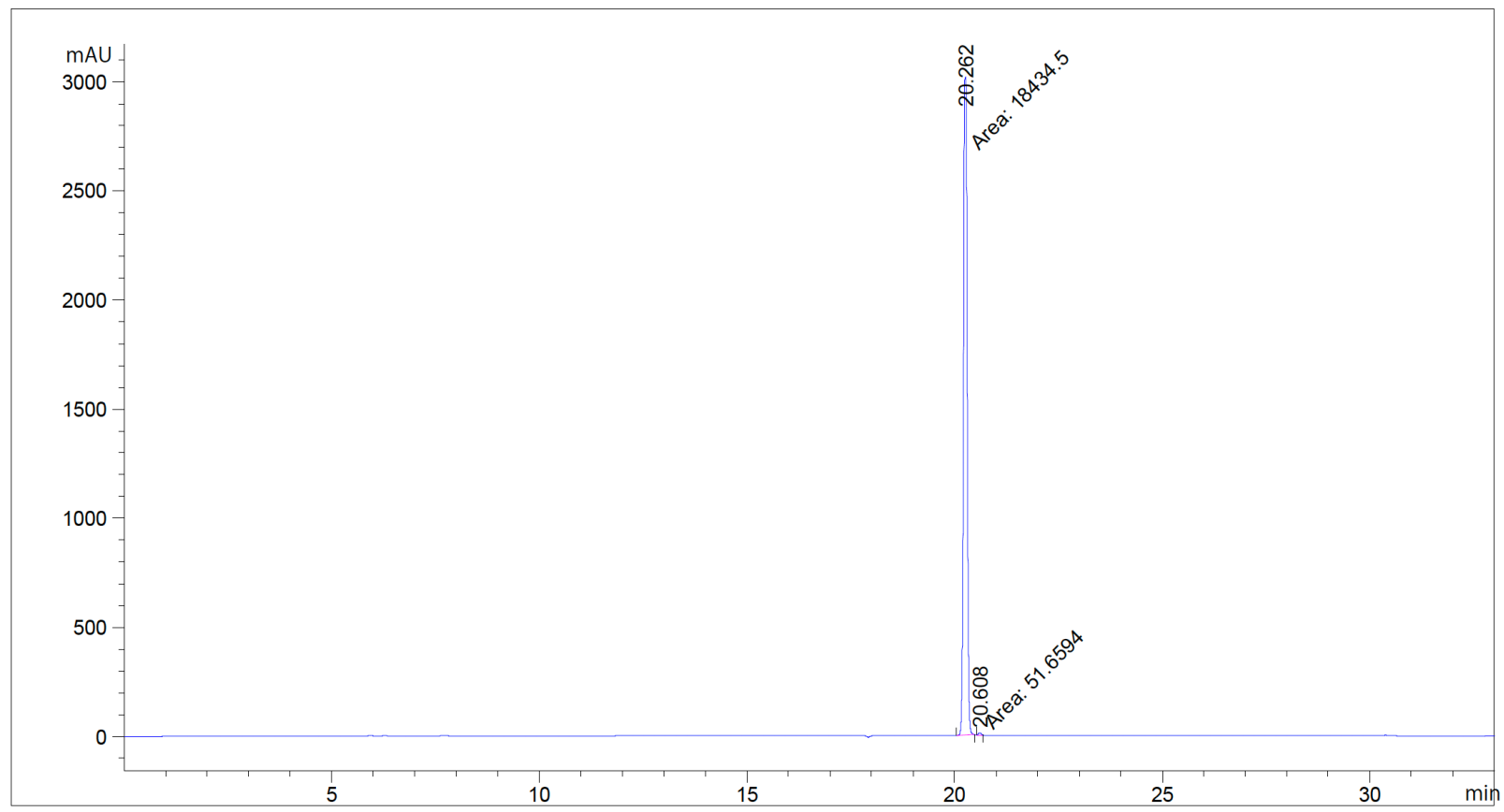

Signal 1: DAD1 A, Sig=260,4 Ref $=360,100$

Peak RetTime Type Width Area Height Area

\# [min] [min] $\left[\mathrm{mAU}^{*} \mathrm{~s}\right][\mathrm{mAU}] \%$

-

120.262 MM $\quad 0.10191 .84345 \mathrm{e} 4 \quad 3014.4677799 .7206$

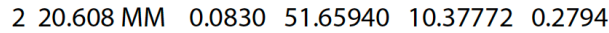

Totals : $\quad 1.84862 \mathrm{e} 4 \mathbf{3 0 2 4 . 8 4 5 5 0}$ 
Fig. S5 HPLC trace for Y3<smiles>O=[N+]([O-])c1ccc(NS(=O)(=O)c2cc(Cl)ccc2Cl)c2ccccc12</smiles>

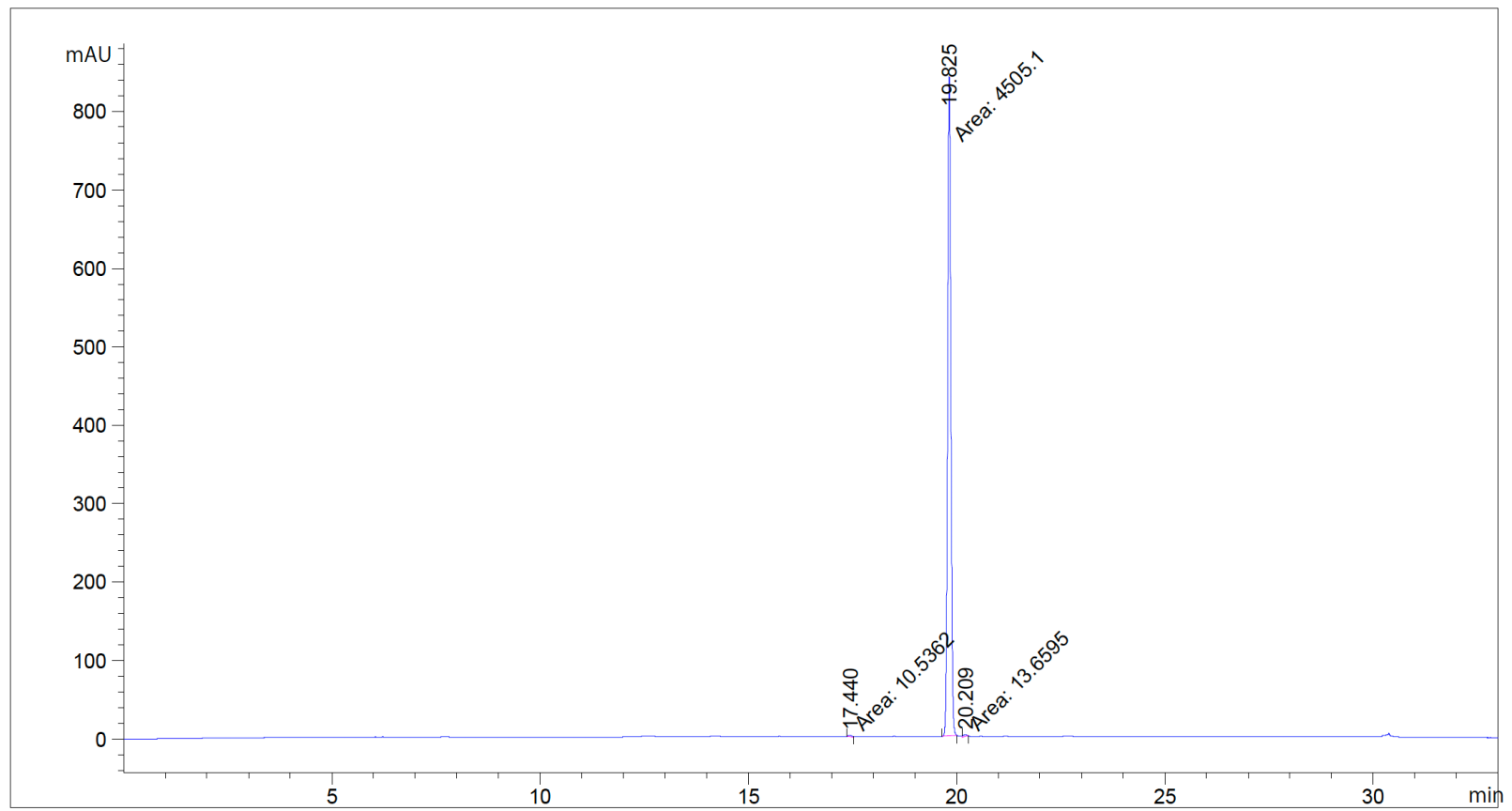

Signal 1: DAD1 A, Sig $=260,4$ Ref $=360,100$

Peak RetTime Type Width Area Height Area

\# [min] [min] $\left[\mathrm{mAU}^{*} \mathrm{~s}\right] \quad[\mathrm{mAU}] \%$

|

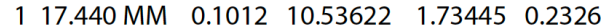

$2 \quad 19.825$ MM $\quad 0.08944505 .09961840 .2323099 .4658$

$\begin{array}{llllll}3 & 20.209 \text { MM } & 0.0996 & 13.65953 & 2.28461 & 0.3016\end{array}$

Totals : $\quad 4529.29536844 .25137$ 
Fig. S6 HPLC trace for Y3-M<smiles>CN(c1ccc([N+](=O)[O-])c2ccccc12)S(=O)(=O)c1cc(Cl)ccc1Cl</smiles>

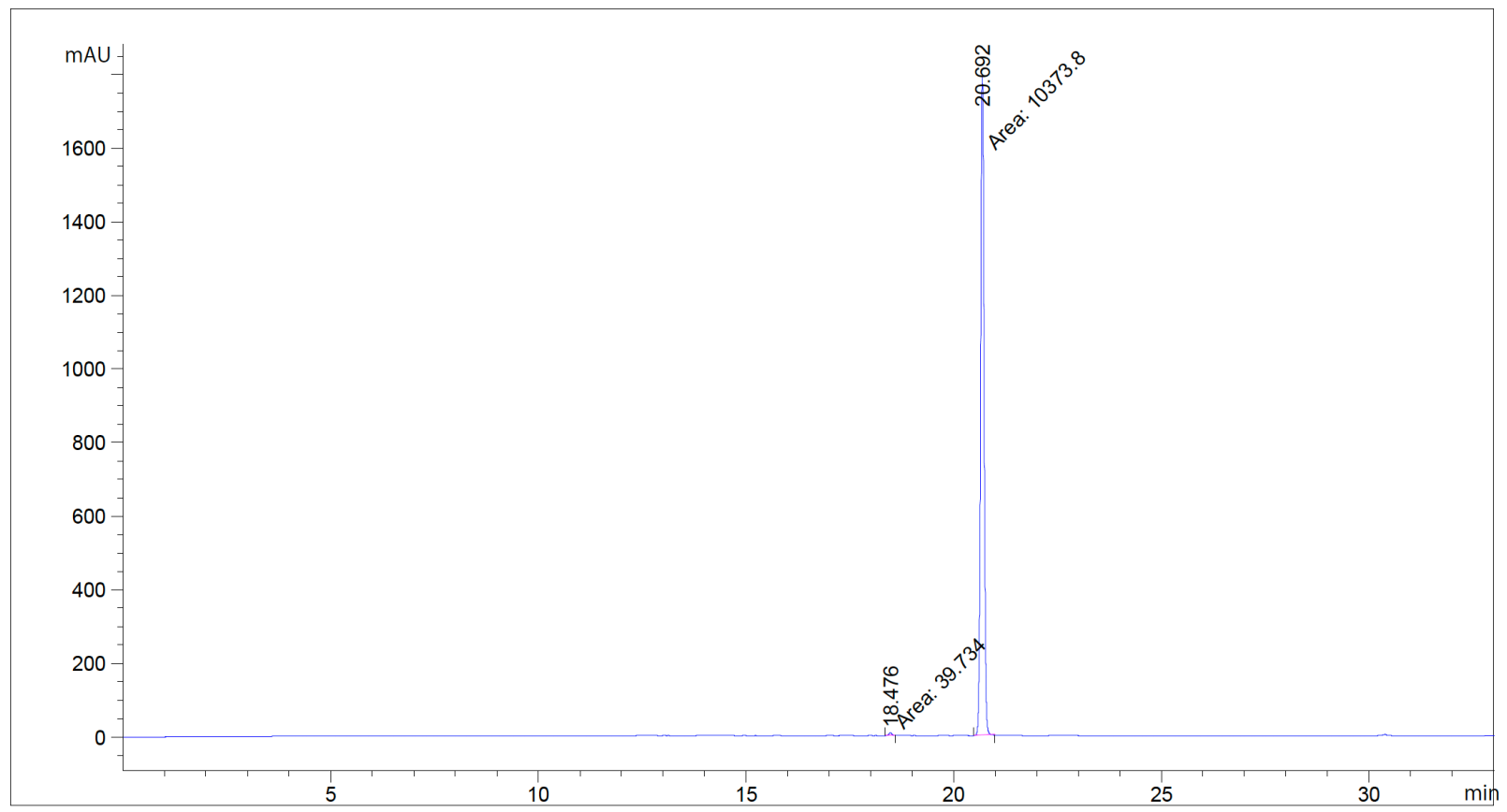

Signal 1: DAD1 A, Sig=260,4 Ref $=360,100$

Peak RetTime Type Width Area Height Area

\# [min] [min] $\left[\mathrm{mAU}^{*} \mathrm{~s}\right] \quad[\mathrm{mAU}] \%$

|

$\begin{array}{llllll}1 & 18.476 \text { MM } & 0.0886 & 39.73402 & 7.47361 & 0.3816\end{array}$

2 20.692 MM $\quad 0.09671 .03738 \mathrm{e} 4 \quad 1787.2749099 .6184$

Totals : $\quad 1.04135 \mathrm{e} 41794.74851$ 